\title{
Avaliação do uso do Hylano GF-20 no pós-operatório de artroscopia de joelho por artrose
}

\author{
The evaluation of Hilan GF-20 in the postoperative knee arthroscopies for arthrosis
}

\author{
Wilson Abou Rejalli' ${ }^{1}$ Alceu Gomes Chueire ${ }^{2}$, José Antonio Cordeiro ${ }^{3}$, \\ Flavio Calil Petean ${ }^{4}$, Guaracy de Carvalho Filho ${ }^{5}$
}

\section{RESUMO}

O presente estudo avaliou os benefícios do uso do Hylano GF-20 no pós-operatório de artroscopias do joelho para lavagem e desbridamento por artrose.Foram estudados 20 pacientes submetidos à artroscopia em 20 joelhos que apresentavam artrose até grau 3 da classificação com sintomas de dor e bloqueio no referido joelho e não obtiveram melhora ao tratamento conservador, sendo então realizada lavagem associada a desbridamento leve condral e meniscal de acordo com a necessidade de cada caso. Os pacientes foram divididos em 2 grupos sendo que em um dos grupos foi feita apenas a artroscopia e noutro grupo artroscopia associada ao uso de 3 infiltrações realizadas semanalmente de Hylano GF-20.Os pacientes foram avaliados nas variáveis dor em repouso noturna, dor durante movimento com sobrecarga de $10 \%$ do peso corporal, dor durante o movimento mais doloroso do joelho afetado com escala visual e a variável quantidade diária de diclofenaco potássico ingerida para alívio da dor no joelho afetado.

Os resultados estatísticos mostraram melhora significativa em todas as variáveis estudadas nos pacientes do grupo submetidos ao uso de infiltrações com o Hylano GF-20 no pós-operatório de artroscopias do joelho por artrose no período de 6 meses.

Descritores: Joelho; Artrose; Artroscopia.

\section{SUMMARY}

This study evaluated the benefits of the Hylano GF-20 use in the postoperative period of knee arthroscopies for irrigation and debridement due to arthrosis. Twenty knees of twenty patients underwent arthroscopy. They presented arthrosis up to $3^{\text {rd }}$ classification degree, pain and had their knees blocked. The segmental treatment showed no improvement, consequently irrigation associated with mild condral and meniscus debridement was performed according to each case.

The patients were divided into two groups: arhtroscopy was performed in one; in the other, arthroscopy associated with the use of three weekly infiltrations of Hylano GF-20. The patients were evaluated according to the following variables: pain during night sleep; pain during movements with loads of $10 \%$ of the body weight; pain during more severe movements of the injured knee with visual scale and the daily amount of potassium diclofenac ingested to relieve the pain of the injured knee. The statistical results showed a significant improvement in relation to all studied variables of patients from the group submitted to the use of Hylano GF-20 infiltration in the postoperative period of knee arthroscopy due to arthrosis.

Keywords: Knee; Arthrosis; Arthroscopy.

\section{INTRODUÇÃO}

A osteoartrose é a doença degenerativa articular mais prevalente, resultando em significativa morbidade com um dos mais altos custos de tratamento para os sistemas de saúde no mundo todo ${ }^{(1,7,12,13)}$. O processo de adoecimento é caracterizado por progressiva erosão da cartilagem articular, levando à diminuição do espaço articular, esclerose subcondral, formação de osteofitos marginais, cistos subcondrais e inflamação sinovial resultando em dor e diminuição da função, levando estes paciente à procura de cuidados médicos ${ }^{(1,2,12)}$.

A cartilagem normal é um tecido avascular formado por uma grande matriz extracelular e esparsamente povoada de células. A água representa 66 a $80 \%$ de sua estrutura e o material orgânico é composto de 48 a $62 \%$ de colágeno tipo II e de 22 a $38 \%$ de proteoglicanos. Esse conjunto de líquidos de densidade variável, fibras com direções diversas e células dispostas de per- meio é nutrido por embebição, e o seu processo anabólico e catabólico é regido por diversos fatores, em especial os de crescimento. A homogeneidade e o equilíbrio dessa complexa estrutura são mantidos por diversas enzimas, na sua maioria secretada pelo condrócito e por células sinoviais. No processo de artrose há desagregação desse equilíbrio e a desestruturação da cartilagem ocorre pela sua fragmentação, que resulta na liberação de enzimas degradadoras da matriz pelos condrócitos, levando inicialmente à fibrilação e fragmentação e, finalmente ocorrem as lesões na superfície articular como abaulamentos e ulceração da cartilagem ${ }^{(2,9)}$.

A artrose do joelho pode ser classificada em primária (idiopática) ou secundária à patologias inflamatórias, infecciosas ou traumáticas que destroem a estrutura da cartilagem ${ }^{(2,9)}$. O tratamento clássico inicial da osteoartrose do joelho usualmente foi com o uso de drogas analgésicas e antiinflamatórias, injeções intrarticulares de corticosteróides associadas à fisioterapia e

Trabalho realizado no Departamento de Ortopedia e Traumatologia da Faculdade de Medicina de São José do Rio Preto - SP - FAMERP-FUNFARME.

1. Mestrando pela FAMERP

2.Chefe do Departamento de Ortopedia e Traumatologia

3.Livre Docente pela UNESP, Coordenador de Ensino I no Departamento de

Epidemiologia e Saúde Coletiva

4.Médico Assistente da Disciplina de Imunologia Clínica do Departamento de Clínica

Médica

5.Chefe da Disciplina de Ortopedia e Traumatologia da FAMERP-FUNFARME
Endereço para correspondência: Rua Campos Sales 1767 - CEP: 15025-600

São José do Rio Preto-SP.

e-mail: wilsonrejaili@unimedriopreto.com.br 
perda de peso. Com o conhecimento melhor, porém, parcial da etiopatogenia, recentemente novas drogas têm sido estudadas e usadas com relativo sucesso, visando proteção da estrutura da cartilagem como é o caso da diacíreina, sulfato de glicosamina e condroitina e os óleos insaponificáveis que parecem atuar, diminuindo a ação das enzimas degradadoras e protegendo a cartilagem articular.

Sabe-se que um elemento também importante na fisiopatologia da osteoartrose é a perda das propriedades viscoelásticas do líquido sinovial e, assim, foram desenvolvidos os hialuronatos como tratamento conservador, eficaz nas artroses através de infiltração destes fármacos por via intrarticular(2, 7,9,12,13).

Com a falha no tratamento conservador podemos realizar vários tipos de tratamentos cirúrgicos como artroscopia para lavagem e desbridamento articular, osteotomias corretivas e artroplastia parcial ou total do joelho dependendo do grau da artrose $^{(1,2,12)}$.Certamente o mais polêmico destes tratamentos relatados na literatura seja a lavagem associada ao desbridamento articular. "Lavar" a articulação do joelho diminui a concentração das enzimas líticas, remove os detritos da cartilagem articular e os corpos livres, uma fonte teórica de sinovite persistente, e inclui a realização apropriada de meniscectomias que levem a bloqueios e dor.Historicamente os desbridamentos foram descritos pela primeira vez por Pridie appud Scott ${ }^{(10)} \mathrm{e}$ Magnunson appud $\operatorname{Scott}^{(10)}$ e mais tardiamente foram analisados por Insall appud Scott ${ }^{(10)}$. Pridie appud Scott ${ }^{(10)}$ encontrou $64 \%$ de êxito e Magnunson appud $\mathrm{Scott}^{(10)}$ descreveu uma resposta fibrocartilaginosa presente após o desbridamento leve das lesões cartilagíneas.Além destes temos resultados satisfatórios encontrados por Rand appud $\operatorname{Scott}^{(10)}(74 \%)$, Sprague appud Scott ${ }^{(10)}$ (75\%), Bert e Macshka appud Scott ${ }^{(10)}$ (77\%) que associados aos quase 500 casos de Insall appud Scott ${ }^{(10)}$, levaram este a conclusão após uma revisão retrospectiva, que a lavagem e desbridamento quando o paciente apresenta sintomas de travamento mecânicos estariam indicados. Usualmente, o Hylano GF-20 (hialuronato de alto peso molecular) tem sido usado por cirurgiões ortopedistas por meio de infiltração intrarticular no pós-operatório de artroscopias por artrose do joelho.Este procedimento é feito com base no fato do líquido sinovial na artrose do joelho estar com as propriedades de viscosidade e elasticidade diminuídas e, em trabalhos prévios deste fármaco para artrose no tratamento conservador mostrando sua eficácia ${ }^{(2,7,912,13)}$.

O objetivo desta pesquisa foi avaliar, se há, benefícios no uso deste fármaco no pós-operatório de artroscopias para lavagem e desbridamento.

\section{CASUÍSTICA E MÉTODO}

Foram estudados 20 pacientes, portadores de artrose do joelho refratária a tratamento conservador com sintomas de bloqueio e dor. Eram do Ambulatório de Ortopedia da Irmandade da Santa Casa de Misericórdia de São José do Rio Preto - SP e foram submetidos a tratamento artroscópico para lavagem e debridamento articular.

O estudo foi randomizado e os pacientes foram divididos em 2 grupos no período de setembro de 2002 até março de 2003.

No grupo I (10 pacientes), foi estudado o comportamento do tratamento com artroscopia (lavagem e debridamento articular) associada à infiltração pós-operatória semanal em número de três de Hylano GF-20.Nove pacientes eram do sexo feminino e 1 do sexo masculino, com idade variando de 44 a 57 anos (média de 52,80 anos). Do total, sete apresentavam afec- ção do lado direito e três do lado esquerdo, sendo que todos os pacientes eram portadores de artrose grau 3 de Kellgren e Lawrence $^{(5)}$ ao exame radiográfico.

No grupo II(10 pacientes), foi estudado o comportamento do tratamento com artroscopia (lavagem e debridamento articular) sem uso de qualquer medicação condroprotetora pósoperatória, sendo sete pacientes do sexo feminino e 3 pacientes do sexo masculino com idade variando de 39 a 73 anos (média $=56,10$ anos). Do total de 10 joelhos tratados, seis apresentavam afecção do lado direito e quatro do lado esquerdo, sendo que todos os pacientes eram portadores de artrose grau 3 de Kellgren e Lawrence ${ }^{(5)}$ ao exame radiográfico.

No Quadro 1 estão os dados de todos os 20 pacientes estudados.

Os pacientes dos dois grupos foram submetidos a uma avaliação prévia do joelho a ser operado.Foram avaliados os dados da história clínica, exames físico, radiográfico.Também os graus de dor relatada em repouso à noite, dor durante movimento com sobrecarga de $10 \%$ do peso corporal e redução da dor durante os movimentos mais dolorosos do joelho com escala analógica visual.Além destes parâmetros, estudou-se a quantidade de comprimidos de diclofenaco potássico tomado diariamente para alívio da dor no joelho afetado.

No exame pré-tratamento foi fornecido e explicado o Consentimento Informado aprovado pelo Comitê de Ética em Pesquisa da FAMERP (Protocolo n 4119/2002).

Foram apenas estudados os pacientes classificados como grau 3 de Kellgren e Lawrence ${ }^{(5)}$ devido observação prévia na literatura que o fármaco não traz benefícios comprovados em pacientes classificados como grau $4^{(1,18)}$. Abaixo se encontra descrita a classificação de Kellgren e Lawrence ${ }^{(5)}$ em seus 5 graus:

Grau 0 - Nenhuma alteração radiográfica característica de osteoartrose.

Grau 1 - Presença de mínimo osteofito sem diminuição do espaço articular.

Grau 2 - Presença de mínimo osteofito com mínima diminuição do espaço articular.

Grau 3 - Presença de osteofito moderado com moderada diminuição do espaço articular.

Grau 4 - Espaço articular quase ou

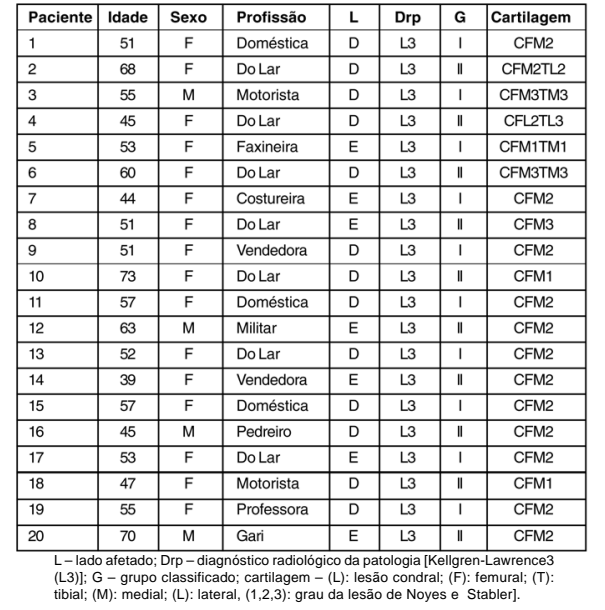

Quadro 1- Dados sobre os 20 pacientes operados. totalmente diminuído com esclerose do osso subcondral.

Os pacientes com grau 3 na classificação radiográfica de Kellgren e Lawren$\mathrm{ce}^{(5)}$ com sintomas de travamento que não melhoraram com tratamento conservador prévio foram, então, submetidos à cirurgia artroscópica para lavagem, debridamento condral e regularização meniscal parcial ou total que se fizessem necessárias. A técnica utilizada foi realizada com três portais (súpero-medial, antero-medial e antero-lateral). O fluido utilizado foi o soro fisiológico a $0,9 \%$ e a regularização cartilagínea meniscal foi feita de maneira suave sem abrasão, exposição ou perfuração do osso subcondral.

Os pacientes foram estimulados a deambular no primeiro dia de pós-operatório e os pontos foram retirados na primeira avaliação com duas semanas. Os pacientes do grupo I foram submetidos a 3 infiltrações semanais de Hylano GF-20 a partir do primeiro retorno; realizadas com botão anestésico(2 $\mathrm{ml}$ de Xilocaína a $2 \%$ sem vasoconstrictor) na pele,assepssia rigorosa na região súpero-lateral do joelho operado. Os pacientes do grupo II não foram submetidos à infiltração com Hylano GF-20.Todos os pacientes foram avaliados com 2,4,8,16 e 24 semanas de pós-operatório usando-se uma escala(régua) analógica vi- 
sual de dor ,onde o lado mostrado ao paciente apresentava faces de dor e cores do azul ao vermelho conforme aumentava a dor, e o lado do observador mostrava o valor dado pelo paciente em números absolutos (dor em repouso à noite,dor durante movimento com sobrecarga de $10 \%$ do peso no joelho afetado e redução de dor durante os movimentos mais dolorosos do joelho). As lesões condrais degenerativas foram classificadas no momento da artroscopia pela classificação de Noyes e Stabler appud Lemark ${ }^{(6)}$ como grau l: lesão tipo condromalácia, grau II: lesão da cartilagem sem visualização do osso subcondral e grau III: exposição óssea.

A análise estatística foi efetuada no Laboratório para Delineamento de Estudos e Análises de Dados do Departamento de Epidemiologia e Saúde Coletiva da Faculdade de Medicina de São José do Rio Preto.

Os dados seguiram a distribuição normal conforme mostraram os gráficos, o que foi verificado pelo teste de Anderson-Darling (Figura 1):

Para a comparação de 2 médias foi utilizado o teste $t$ de Welch.

Para comparação efeito de grupo, de tempo e interação grupo x tempo, foi utilizada a análise de variância para medidas repetidas. $\mathrm{O}$ uso deste teste só foi possível devido os dados dos grupos terem seguido distribuição normal.

Foi adotado o nível de significância $\alpha=0,05$, por ser usual em ciências da saúde.

\section{RESULTADOS}

Foram encontrados tanto no grupo I (9) como no grupo II(7) maior número de pacientes do sexo feminino sendo o valor $p$ encontrado de 0,58 e, portanto não houve evidência de diferença de proporção de mulheres entre os grupos estudados.

A média de idade foi encontrada no grupo I foi de 52,80 anos e no grupo II, 56,10. Portanto não houve evidência de diferença de idade média entre os grupos estudados (valor- $p=0,43$ ).

Avaliando os resultados encontrados de todas as variáveis no pré-operatório observou-se que não houve evidência de diferença de médias no pré-operatório (Tabela 1).

Avaliando os resultados encontrados de todas as variáveis na primeira avaliação, observou-se que não houve evidência de diferença de médias na primeira avaliação, conforme (Tabela 2).

Avaliando os resultados encontrados de todas as variáveis na primeira avaliação, observou-se que não houve evidência de diferença de queda de médias da avaliação pré-operatória até a primeira avaliação pós-operatória (Tabela 3).

Avaliando os resultados encontrados de todas as variáveis desde a primeira à quinta avaliação, observou-se que houve evidência de queda no grupo I e de estabilidade nas variáveis dor em repouso, dor com carga e dor ao movimento mais doloroso e aumento na variável diclofenaco no grupo // desde a primeira à quinta avaliação pós-operatória (Tabela 4).
Avaliou-se o grau de lesão de cartilagem encontrada nas artroscopias em relação às médias encontradas; observou-se que não seria fator limitante à melhora encontrada no grupo I, (Figuras 2 a 5:)

\section{DISCUSSÃO}

As lesões da cartilagem articular são consideradas o maior problema da ortopedia atual. No caso da artrose do joelho especificamente, grande divergência existe sobre qual seria o meIhor tratamento quando o conservador, falha principalmente nos casos de artrose ainda em estágios não graves, pois nestes a osteotomia ou a artroplastia estariam indicadas. O grande problema destes tratamentos além do custo são os graves riscos existentes, desde os clínicos até os do implante a serem utilizados, trazendo complicações para os pacientes. Grande divergência existe na literatura, mas a artroscopia associada à lavagem

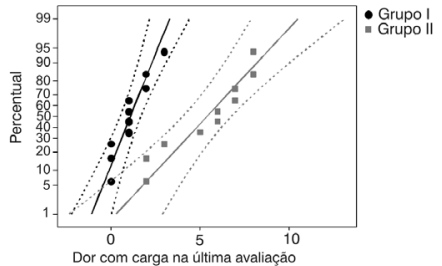

Dor com carga na útima avaliaçáo de dor durante movimento com ndo comportamento normal das

i utilizado o teste $t$ de Welch. e desbridamento leve ainda tem sido usada com grande freqüência e com bons resultados em todo o mundo ${ }^{10}$.

O advento das drogas condroprotetoras (diacíreina, sulfato de glicosamina e condroitina, óleos insaponificáveis e hialuronatos) traz uma nova possibilidade de melhora no resultado final do tratamento artrocóspico. A idéia da inibição da produção de interleucina-1 impedindo que metaloproteases destrua a matriz cartilaginosa evitaria por algum tempo a necessidade de correção de instabilidades ou desvios de eixos mecânicos tornando os tratamentos menos agressivos, diminuindo substancialmente os riscos cirúrgicos.

Sabendo-se previamente que um elemento importante na fisiopatologia da osteoartrose é a perda das propriedades viscoelásticas do líquido sinovial devido à diminuição da concentração de ácido hialurônico e do seu tamanho molecular, e isto aumenta consideravelmente a susceptibilidade da cartilagem às lesões por sobrecarga; o uso dos hialuronatos no pós-operatório de artroscopia para lavagem e desbridamento leve passou a ser uma possibilidade também de melhora dos resultados devido à capacidade dos hialuronatos em diminuir o vetor de pressão causado pelo peso; proteger a integridade da cartilagem articular; estabilizar a matriz cartilagínea pela inibição de perda de proteoglicanas e conseguir intervir, assim, a evolução do processo de destruição tecidual melhorando o resultado pósoperatório do referido tratamento cirúrgico ${ }^{(1,2,9,12)}$.

Na literatura mundial observou-se uma certa tendência à utilização do Hylano GF-20, devido ao seu maior peso molecular, pois teria um poder de absorção de peso maior que os de baixo peso molecular, com melhores resultados em relação à melhora da dor e capacidade funcional do joelho(1, 9,12,13). Além desta propriedade física, acredita-se que o Hylano GF-20 também apresente ainda outras propriedades teórica que auxiliariam na melhora da dor e da capacidade funcional do joelho com artrose descritas por Watterson e Esdaile ${ }^{(12)}$ em seu artigo:

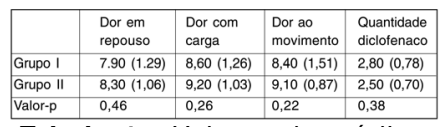

Tabela 1 - Valores de médias, desvio padrão (dp) e valores-p de todas as variáveis na avaliação do pré-operatório.

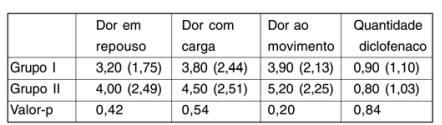

Tabela 2 - Valores de médias, desvio padrão (dp) e valores-p de todas as variáveis na primeira avaliação do pós-operatório.

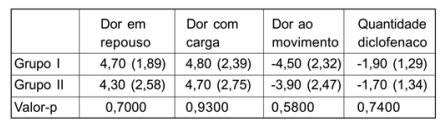

Tabela 3 - Valores de médias, desvio padrão (dp) e valores-p de todas as variáveis da avaliação pré-operatória até a primeira avaliação pós-operatória.

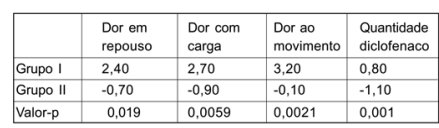

Tabela 4 -Valores de médias e valores- $p$ de todas as variáveis desde a primeira à quinta avaliação pós-operatória. 


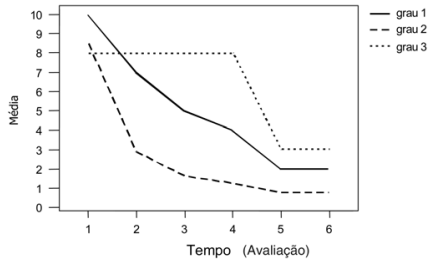

Figura 2 - Variável dor durante movimento de $10 \%$ do peso corporal no grupo I em relação ao grau de lesão da cartilagem encontrada na artroscopia.

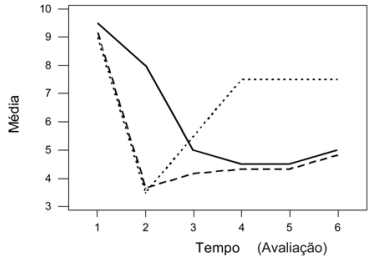

Figura 3 - Variável dor durante movimento de $10 \%$ do peso corporal no grupo II em relação ao grau de lesão da cartilagem encontrada na artroscopia.

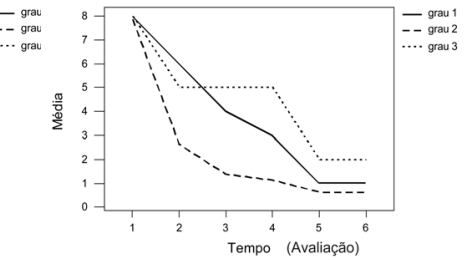

Figura 4 - Variável dor em repouso à noite no grupo I em relação ao grau de lesão da cartilagem encontrada na artroscopia.

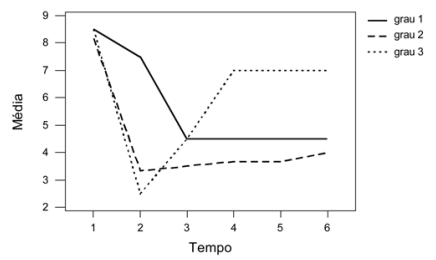

Figura 5 - Variável dor em repouso à noite no grupo II em relação ao grau de lesão da cartilagem encontrada na artroscopia.

-Propriedade antiinflamatória: $\bigcirc$ ácido hialurônico está envolvido na transdução dos sinais dos receptores de superfície das células leucocitárias e que são dependentes do tamanho da molécula do ácido hialurônico e, então, a administração do mesmo de forma intrarticular reduziria os níveis dos mediadores inflamatórios.

- Atividade analgésica: Estudos em modelos experimentais utilizando ratos e gatos, mostraram redução da atividade nociceptiva em articulações inflamadas, talvez por ação direta na sinóvia ou indiretamente através da substância P.

A idéia de estudar o comportamento do uso de Hylano GF20 no pós-operatório de artroscopias de joelho para lavagem e desbridamento, deveu-se ao uso contínuo por cirurgiões de joelho de maneira empírica e sem comprovação científica.Na tentativa de encontrar apoio na literatura, foram encontrados apenas dois trabalhos que suportassem que o uso de hialuronatos de baixo ou alto peso moleculares pudesse ser importante no pós-operatório de artroscopias do joelho, melhorando o resultado pós-operatório das mesmas.

Vad et al(11) testaram lavagem do joelho com soro fisiológico (realizada em consultório) previamente à infiltração de Hylano GF-20, com um grupo controle apenas com infiltrações de Hylano GF-20 isoladamente, para pacientes com artrose de joelho e observou melhores resultados em relação à dor e capacidade funcional nos pacientes submetidos à lavagem prévia em relação ao grupo em que o Hylano GF-20 foi utilizado sozinho.

Daí et al ${ }^{(3)}$ realizaram várias infiltrações de hialuronato de sódio semanais no pós-operatório de artroscopias cirúrgicas do joelho e encontrou que o uso de hialuronato no pós-operatório de cirurgia do joelho parecia eficaz no alívio da dor pós-operatória das referidas cirurgias.

Em relação aos resultados encontrados em nosso estudo, observou-se que não houve diferença entre os dois grupos em relação ao sexo e em relação à média de idade que estava em concordância com a literatura mundial(2,9).

Os resultados estatísticos encontrados na avaliação pré-operatória mostraram que os grupos não tinham diferença estatísti-

ca em relação às quatro variáveis estudadas, o que mostrou a homogeneidade dos dois grupos estudados.

Em relação ao tratamento artroscópico não se encontrou nenhuma complicação intra ou pós-operatória nos pacientes estudados.

Em relação à sinovite pós-infiltração, complicação mais comum encontrada na literatura com o uso de Hylano GF-20 , variando de $11 \%$ encontrada por Puttick et a ${ }^{(8)}$ até $47 \%$ por Henderson et al(4), no estudo realizado esta complicação não foi observada.

Em relação à análise isolada da primeira avaliação pós-operatória, observou-se queda de todas as variáveis estudadas em ambos os grupos demonstrando que o tratamento cirúrgico provavelmente era o fator diferencial na melhora das variáveis estudadas, pois até aquele momento as infiltrações com Hylano GF20 não haviam sido realizadas. Esta análise é semelhante à literatura relata haver melhora com a realização da lavagem e desbridamento leve através da artroscopia.

Nas avaliações estatísticas, nas quais se estudou o comportamento das variáveis após ou com o uso do Hylano GF-20, observou-se sempre melhora ou tendência à queda das médias no grupo I em relação ao grupo II.

Os dados encontrados sugeriram que o grau de lesão da cartilagem (de I a III de Noyes e Stabler) não seria fator limitante para a melhora observada, pois se observou sempre melhora ou tendência à queda no grupo I em relação ao grupo II.

Levando-se em consideração os dados estatísticos, observou-se que o Hylano GF-20 foi o diferencial da melhora das variáveis estudadas em relação aos dois grupos e, também que seu uso rotineiro no pós-operatório de artroscopia no tratamento da artrose pode melhorar o resultado final deste tratamento no período de seis meses.

\section{CONCLUSÃO}

O uso do Hylano GF-20 no pós-operatório de artroscopia para lavagem e desbridamento articular leve por artrose, resultou na melhora da dor e capacidade funcional dos joelhos dos pacientes operados no período de seis meses.

\section{REFERÊNCIAS BIBLIOGRÁFICAS}

1. Adams ME,Atkinson MH,Lussier AJ,Schulz JI,Siminovitch KA, Wade JP et al. The role of viscosupplementation with hylan G-F 20 in the treatment of osteoarthritis of the knee: a Canadian multicenter trial comparing hylan G-F 20 alone, hylan G-F 20 with non-steroidal anti-inflammatory drugs(NSAIDs) and NSAIDs alone.Osteoarthritis and Cartilage 3:213-225,1995.

2. Camanho GL. Tratamento da osteoartrose do joelho.Rev Bras Ortop 36: 135-140,2001.

3. Dai GF, Tang JW, Wang SJ, Liu Q, Shi DP. Zhongguo Xiu Fu Chong Jian Ke Za Zhi 16: 8-16,2002.

4. Henderson EB, Grootveld M, Smith EC, Thompson PW, Blake DR.Ann Rheum Dis 50: 196-200, 1991.

5. Kellgren JH, Lawrence JS.Radiological assessment of osteoarthrosis.Ann Rheum Dis 16:494-501, 1957.

6. Lemark LJ,Marushack MM. Tratamento da Cartilagem Articular.In:Andrews JR, Timmerman LA,Editores.Artroscopia:Diagnóstico e Cirurgia. $1^{\text {a }}$ ed.londres:Chapman and Hall/ CRC;1999.p.189-97.

7. Pelletier JP, Pelletier JM. The Pathophysiology of Osteoarthritis and the Implication of the Use of Hyaluronan and Hylan as Therapeutic Agents in Viscosupplementation. J Rheumatol 20:19-24,1993.

8. Puttick MPE, Wade JP, Chalners A,Connell DG, Rangno KK.Acute Local Reactions after Intraarticular Hylan for Osteoarthritis of the Knee.J Rheumatol 22:1311-1314,1995.

9. Rezende MU. Efeito do Ácido Hialurônico e da Diacíreina na Artrose: Modelo Experimental em Ratos [dissertação]. São Paulo (SP): Faculdade de Medicina da Universidade de São Paulo, 2000

10. Scott WN, Insall JN, Kelly MA.Artroscopia Y meniscectomía: abordaje, anatomia y técnicas quirúrgicas. In: Insall JN.Cirugía de la Rodilla. $2^{a}$ edição. Buenos Aires: Editora Medica Panamericana,1994. p 165-225.

11. Vad VB,Bhat AL, Sculco TP,Wickiewicz TL. Management fo the Knee Osteoarthritis : Knee Lavage Combined With Hylan Versus Hylan. Alone.Arch Phys Rehabil 84:634-637,2003.

12. Watterson JR, Esdaile JM. Viscosupplementation: Therapeutic Mechanisms and clinical Potential in Osteoarthritis of the Knee. J Am Acad Orthop Surg 8: 277-284,2000.

13. Wobig M, Bach G, Beks P, Dickhut A, Runzheimer J,Schwieger $G$ et al. The Role of Elastoviscosity in the Efficacy of Viscosupplementation for Osteoarthritis of the Knee: A Comparison of Hylan G-F 20 and Lower-Molecular-Weight Hyaluronan.Clin Ther 21: 1549-1562,1999. 